Cinémas

Revue d'études cinématographiques

Journal of Film Studies

\title{
La sublimation comme formation de la forme. Notes sur un article inédit d'Eisenstein
}

\section{Mikhaïl Iampolski}

Volume 11, numéro 2-3, printemps 2001

Eisenstein dans le texte

URI : https://id.erudit.org/iderudit/024849ar

DOI : https://doi.org/10.7202/024849ar

Aller au sommaire du numéro

Éditeur(s)

Cinémas

ISSN

1181-6945 (imprimé)

1705-6500 (numérique)

Découvrir la revue

Citer cet article

Iampolski, M. (2001). La sublimation comme formation de la forme. Notes sur un article inédit d'Eisenstein. Cinémas, 11(2-3), 111-144.

https://doi.org/10.7202/024849ar
Résumé de l'article

Cet article introduit un texte d'Eisenstein resté inédit, « Rayon et Gnôle » écrit au printemps 1925, après la réalisation de La Grève et avant la conception de Potemkine. C'est une première prise de position d'Eisenstein sur la psychanalyse en tant que méthode artistique et critique. Le problème qu'il tend à résoudre est celui de la forme, qu'il a abordé à cette époque dans « Sur la question d'une approche matérialiste de la forme ». Ici, Eisenstein critique deux films récents, Le Rayon de la mort du collectif Kouléchov et La Course à la gnôle d'Abram Room. Kouléchov y est décrit comme un stylisateur dont les films ont une forme trop figée, immobile, non organique. Room, au contraire, totalement incapable de produire la forme, s'enlise dans l'informe. Eisenstein oppose à Kouléchov et à Room sa propre approche d'une forme artistique fondée sur la sublimation d'une perversion sexuelle. La " meilleure» perversion pour un réalisateur est un sadisme refoulé (Eisenstein cite Stroheim et Griffith comme exemples). 


\section{La sublimation comme formation de la forme. Notes sur un article inédit d'Eisenstein}

\section{Mikhail Iampolski}

\section{RÉSUMÉ}

Cet article introduit un texte d'Eisenstein resté inédit, "Rayon et Gnôle» écrit au printemps 1925, après la réalisation de La Grève et avant la conception de Potemkine. C'est une première prise de position d'Eisenstein sur la psychanalyse en tant que méthode artistique et critique. Le problème qu'il tend à résoudre est celui de la forme, qu'il a abordé à cette époque dans "Sur la question d'une approche marérialiste de la forme m. Ici, Eisenstein critique deux films récents, Le Rayon de la mort du collectif Kouléchov et $L a$ Course à la gnôle d'Abram Room. Kouléchov y est décrit comme un stylisateur dont les films ont une forme trop figée, immobile, non organique. Room, au contraire, totalement incapable de produire la forme, s'enlise dans l'informe. Eisenstein oppose à Kouléchov et à Room sa propre approche d'une forme artistique fondée sur la sublimation d'une perversion sexuelle. La "meilleure" perversion pour un réalisateur est un sadisme refoulé (Eisenstein cite Stroheim et Griffith comme exemples).

\section{ABSTRACT}

This article introduces one of Eisenstein's unpublished texts, "Rays and Moonshine", written in spring 1925 between the making of Strike and the conception of Potemkin. It represents an initial foray by Eisenstein into psychoanalysis as an artistic and critical method. The article's subject is film form, taken up elsewhere in this period in "On the Question of a Materialist 
Approach to Form". Here, Eisenstein critiques two recent films, The Death Ray by the Kuleshov collective and The Race for Moonshine Vodka by Abram Room. Eisenstein describes Kuleshov as a stylist whose films have a form which is too congealed, immobile, and inorganic. Room, however, is seen as completely incapable of producing a form. To Kuleshov and Room Eisenstein opposes his own approach, that of an artistic form founded on the sublimation of a sexual perversion. For Eisenstein, the "best" perversion a filmmaker can have is that of a suppressed sadism, and he cites Stroheim and Griffith as examples.

L'article d'Eisenstein «Rayon et Gnôle [Essai de définition de la carence idéologique dans le domaine de la forme) " (désormais " $R G »)$ est un chaînon très important dans le développement de l'esthétique eisensteinienne. En raison de son contenu choquant et de la non-orthodoxie de ses conclusions, cet article est resté dans les archives plus de soixante-dix ans. Son importance tient en premier lieu à ce qu'il se rapporte à la première période de création du réalisateur, quand les fondements théoriques de sa méthode n'ont toujours pas été définitivement formulés. "RG " permet d'examiner la logique de la pensée eisensteinienne immédiatement après la formulation des principes fondamentaux $\mathrm{du}$ "montage des attractions».

Commençons par le contexte chronologique immédiat de l'article.

« RG» a été terminé le 2 avril 1925. Ce travail coïncide avec l'achèvement de La Grève (projection publique le 9 mars, sortie en salle le 28 avril 1925) et avec l'abandon du projet inachevé Cavalerie rouge d'après Isaac Babel. Le $1^{\text {er }}$ avril, le département moscovite de Sevzapkino, qui avait accepté la réalisation de $\mathrm{Ca}$ valerie rouge, a été dissous.

Dans le domaine de la théorie, Eisenstein se trouve toujours à l'intérieur de la problématique de l'attractionnisme. Son texte "Le montage des attractions au cinéma", qui date d'octobre 1924, paraît dans le livre d'Alexandre Belenson, Le Cinéma aujourd'hui, mutilé par l'éditeur. Au printemps 1925, Eisenstein écrit deux articles qui contiennent l'analyse détaillée de nouveaux films soviétiques. L'un est "RG", qui a été écrit immédia- 
tement après deux sorties: Course à la gnôle d'Abram Room (le 5 mars 1925) et Le Rayon de la mort de Lev Kouléchov (le 16 mars 1925 ). Le deuxième article, "Sur la question d'une approche matérialiste de la forme", est construit sur la confrontation de l'esthétique de La Grève avec celle du Kinoglaz de Vertov (sorti le 13 octobre 1924). Ce deuxième article a été publié dans le Kino journal $A R K$ et est solidement ancré dans l'usage des historiens de cinéma. " $\mathrm{RG}$ » et "Approche matérialiste» ont beaucoup en commun. Mais si la question de la forme au cinéma se trouve au centre des deux textes, leurs réponses different considérablement. "Approche matérialiste» est beaucoup plus proche par son orientation du "Montage des attractions au cinéma", alors que " $\mathrm{RG}$ " tente d'aller plus loin dans l'interprétation de certaines questions posées par "Le montage des attractions au cinéma ". Quelles sont ces questions?

Dans "Le montage des attractions" - article de 1923 publié dans Lef et qui concerne presque exclusivement le théâtre Eisenstein définit l'attraction comme l'«unité de' l'efficacité au théâtre » et précise :

Est attraction (du point de vue théâtral) tout moment agressif au théâtre, c'est-à-dire tout élément qui fait subir au spectateur une pression sensorielle ou psychologique, vérifiée au moyen de l'expérience et calculée mathématiquement pour produire chez celui qui le perçoit certains chocs émotionnels (1974, p. 117).

Ce bouleversement doit être orienté idéologiquement, mais, sur le plan formel, l'attraction n'est pas du tout définie. L'attraction, en 1923, c'est "tout moment agressif». Et bien qu'Eisenstein ne donne pas de précisions, il choisit cependant de souligner en elle le moment sadique, choquant. L'attraction s'apparente à des techniques proches de celles du théâtre du Grand Guignol

[...] par exemple: yeux crevés ou bras et jambes sectionnés sur scène, participation par téléphone du personnage en scène à un événement cauchemardesque qui se passe à des dizaines de kilomètres de là, ou encore situation de l'ivrogne qui pressent l'approche de la 
mort et dont les appels à l'aide sont pris pour du délire (1974, p. 117).

La base sadique de l'attractionnisme ne s'explique que par son caractère performatif, l'influence qu'elle exerce. Il ne s'agit pas encore d'une forme spécifique. On sait que les films d'Eisenstein sont saturés de scènes sadiques, parfois présentées à la manière du guignol. Quand, dans la première moitié de 1925, c'està-dire en même temps qu'il écrivait " $R G$ ", Eisenstein dicte à Grigori Alexandrov le scénario du Bazar de la concupiscence, il s'amuse et s'emballe dans l'autoparodie. Par exemple (Eisenstein et Alexandrov, 1993b, p. 6):

Sur une pierre tombale (variante: dans la fosse mortuaire), Maria accouche d'un mort. Horreur. Un passant intéressé se glisse dans le bûcher. Au paroxysme du désespoir, Maria fouille la terre avec ses ongles. Le passant arrache le corps de l'enfant à Maria, le secoue en l'air'2.

Ce type d'excès existe en dehors de la problématique de la forme qui intervient dès lors qu'Eisenstein se tourne vers le cinéma, en rapport direct avec La Grève. L'adaptation au cinéma se fait dans "Le montage des attractions au cinéma". L'attraction n'est plus alors une représentation immédiate d'un fait (comme au théâtre), elle agit

par la voie de la confrontation et de l'accumulation dans le psychisme du spectateur des associations nécessaires pour atteindre le but recherché, induites par certains fragments de faits décomposés (pratiquement en "morceaux de montage") et par des associations qui ne donnent qu'ainsi, dans leur ensemble, un tel effet (souvent encore plus puissant). Par exemple, une fois encore, un meurtre: le cou empoigné, les yeux qui montent sur le front, le couteau levé, la victime qui ferme les yeux, le sang qui gicle sur le mur, la victime qui tombe sur le plancher, la main qui essuie le couteau tous fragments destinés à "provoquer" des associations (1974, p. 129-130).

Eisenstein garde à dessein l'exemple de la scène de meurtre sadique, "guignolesque», qui incarne l'agressivité de l'attraction, 
mais il la retourne. L'effet se produit grâce à une chaîne d'associations. D'un côté, la chaîne d'associations oriente la problématique du côté de la réflexologie, de l'autre, du côté du montage et donc, par la problématique du montage, il introduit dans la discussion le problème de la forme. Il se trouve qu'il ne suffit pas au cinéma de montrer «le fait de crever les yeux ou d'amputer les bras et les jambes " comme au théâtre. Ce sont les associations - dont l'attraction produit l'accumulation - qui produisent un effet. C'est pourquoi tout élément agressif doit être décomposé en éléments associatifs. Eisenstein souligne:

Le centre de gravité de l'effet au cinéma, au contraire du théâtre, ne consiste pas dans les influences directement physiques bien qu'il soit possible parfois d'arriver à une captation purement physique (1974, p. 131).

Il se réfere alors à la scène de la fusillade de $L a G_{\text {rève }}{ }^{3}$, célèbre pour sa cruauté, en tant que "bonne" décision cinématographique:

Pour ajouter l'effet maximum d'horreur sanglante [...], j'ai utilisé ceci : une comparaison associative de la fusillade avec un abattoir. La première n'est donnée que par des plans généraux ou moyens "mis en scène" chute de 1500 ouvriers dans le ravin, fuite de la foule, salves, etc. - et tous les gros plans sont dévolus à l'exposition de l'horreur réelle de l'abattoir avec l'égorgement et l'écorchement des bêtes (1974, p. 133).

Eisenstein explique ensuite que c'est un épisode de Mister West, de Kouléchov, qui lui a inspiré l'idée du caractère du montage de l'attraction:

[...] quand un colossal camion tire un petit traineau avec le portefeuille de Mister West. Cette combinaison qu'on trouve en de multiples variantes dans toutes les entrées de clowns excentriques, en commençant par le petit haut de forme et en finissant par les énormes godasses. Sur scène une telle combinaison suffit. Par contre à l'écran où la combinaison est montrée d'un seul coup en un seul cadre (bien qu'au moment de la sortie par le portail, il y ait une petite pause, en raison de la longueur de la corde entre le camion et le traîneau), l'effet produit est très faible. 
Eisenstein oppose à cet échec de Kouléchov la réussite d'« un moment analogue» chez Chaplin

\begin{abstract}
[...] quand un métrage considérable de film est dévolu à l'ouverture infiniment compliquée des serrures d'un énorme coffre-fort, et qu'on découvre plus tard (et il semble même dans un autre plan) un balai, un chiffon et un seau rangés à l'intérieur (1974, p. 130-131).
\end{abstract}

En quoi consiste la différence entre l'approche de Kouléchov et celle de Chaplin? Eisenstein répond: dans la durée d'exposition du coffre-fort et du camion. La durée est nécessaire pour "l'implantation des associations requises". En d'autres termes, si la corde entre la camion et le traîneau chez Kouléchov était plus longue, l'attraction aurait produit son effet. En même temps, Eisenstein s'efforce manifestement de lier l'attraction cinématographique au montage. Son indécision, par rapport à Chaplin, est cependant caractéristique. Il ne se souvient pas précisément si le coffre-fort et les chiffons sont donnés dans le même plan ou non. "Et il semble même dans un autre plan", écrit-il entre parenthèses; visiblement il le souhaite.

Quoi qu'il en soit, dans "Le montage des attractions", la question de la forme du moment agressif se pose encore sur un plan général, sans une élaboration concrète claire. La question principale demeure ainsi inexpliquée: pourquoi l'impact physiologique direct du matériau sadique (car il s'agit précisément de matériau sadique) doit-il être exclu du cinéma? Lappel aux chaînes associatives semble être une manœuvre de diversion, plutôt que la vraie raison. "Sur la question d'une approche matérialiste de la forme " présente visiblement une tentative de réponse mieux articulée à cette question. Il n'est pourtant pas possible de dire qu'Eisenstein a beaucoup avancé. Il est cependant essentiel que la question de l'attraction, c'est-à-dire de l'impact agressif, soit définitivement formulée ici en termes de forme, bien que la compréhension de la forme elle-même reste très floue. Il donne la définition suivante:

La forme révolutionnaire est le produit de procédés techniques correctement établis de concrétisation de la nouvelle vision et de la nouvelle approche de la vie et 
des phénomènes - la nouvelle idéologie de classe vraie rénovatrice non seulement de la signification sociale, mais aussi de la nature matérielle et technique du cinéma qui apparaît dans ledit "notre contenu " (1974, p. 148).

De cette formulation peu claire, il ressort cependant qu'Eisenstein essaie d'interpréter la forme comme la manifestation de l'idéologie dans la combinaison des procédés techniques. La mise au premier plan du "procédé" lui-même renvoie, bien sûr, à la logique du formalisme et va par la suite être rejetée par Eisenstein. En gros, "Approche matérialiste" oppose la structure de montage associatif de La Grève à l'«impressionnisme primitif » amorphe de D. Vertov. L'«impressionnisme» de Vertov est alors interprété, en substance, comme absence de forme, forme à indice négatif. Selon Eisenstein, Vertov

[...] tisse la trame d'un tableau pointilliste des "morceaux de vie véritable", le Kinoglaz, chevalet à la main, court derrière les choses telles qu'elles sont, sans bouleverser violemment l'inévitable statisme de la causalité de leurs enchainements (1974, p. 152).

C'est en raison de l'absence de forme que l'impressionnisme de Vertov ne peut agir activement sur la conscience. Eisenstein rappelle encore une fois la scène de l'abattoir, montrée dans le Kinoglaz et dans La Grève, et affirme qu'elle n'est que "sténographiée" chez Vertov, tandis que dans La Grève elle est "sanglante et impressionnante" (1974, p. 152). L'élément sadique continue de rester au centre de l'attention, mais cette fois il est définitivement affirmé que la puissance de son influence dépend d'une forme correcte.

Eisenstein revient à nouveau sur la question de la forme dans un court article, "La Méthode de réalisation d'un film ouvrier" (août 1925), où il donne enfin la définition de la forme qu'il va fréquemment utiliser par la suite. Il renonce ici à l'aspect technique et formule le concept de forme dans des termes purement psychologiques:

La doctrine des stimuli et de leur montage dans l'orientation définie doit fournir le matériau complet pour la

La sublimation comme formation de la forme. Note sur un article inédit d'Eisenstein 
question de la forme. Le contenu, comme je le conçois, est la liste des bouleversements à enchaîner auxquels on veut soumettre le public... La forme est alors la réalisation de ces mesures sur ce matériau particulier par la voie de la création et de la composition des stimuli mêmes qui peuvent précisément provoquer ce pourcentage nécessaire, c'est-à-dire le côté concrétisant et effectif de l'œuvre (1974, p. 27).

En d'autres termes, le contenu est l'ensemble des stimuli et des moments agressifs ou, comme le dit Eisenstein, des «bouleversements". Et la forme est l'enchaînement concret de ces bouleversements dans tout cas particulier. Ce déplacement vers une psychologisation du concept de forme est extrêmement important, car il déterminera toute la théorisation d'Eisenstein en ce domaine. En 1934, il ébauche une grande série de dessins sur le thème de l'assassinat du roi Duncan dans Macbeth. Le choix même d'un assassinat en tant que motif montre à quel degré cette orientation attractionniste vers le bouleversement agressif était, en 1934 encore, importante pour lui. Dans le bref texte qui analyse ces dessins, il donne une définition de la forme beaucoup plus réfléchie:

La forme est souvent la réflexion dans le matériau du processus psychologique (y compris* l'unité conscientinconscient, individuel-social, intellectuel-émotionnel), du processus créatif propre à l'auteur comme représentant de toute formation sociale (1997/1998, p. 243-244).

La forme est comprise ici comme réflexion dans le matériau du processus psychologique, transfert du processus psychologique dans un autre matériau, à savoir le matériau de l'art.

Le mouvement vers la compréhension psychologique de la forme débute en 1925 et se lie dans la conscience d'Eisenstein avec le travail sur Potemkine. Peu après son achèvement, il décrit ce déplacement dans un article où il affirme que "Potemkine établit une jonction avec une ère nouvelle, celle du nouveau psychologisme" (1974, p. 27). C'est ici que le réalisateur fait une

* Mots, groupe de mots ou expression apparaissant en allemand, anglais, français ou latin dans le texte original russe. 
comparaison entre Potemkine et La Grève qui relève encore de la réflexologie attractionniste réductionniste:

Sur le plan des moyens d'influence, par rapport à $\mathrm{La}$ Grève, Potemkine n'est pas un prolongement mais une négation. À l'absence de sujet, au style de procèsverbal, aux natures mortes de $L a$ Grève [...] est opposé déjà ici un psychologisme dans son plein développement (1974, p. 36).

Pourquoi le concept de forme acquiert-il une telle signification et pourquoi est-il formulé en de tels termes? Cela devient en partie compréhensible à partir de "RG». La forme y est comprise comme produit de l' "appartenance de classe du réalisateur", mais celle-ci est à son tour liée à une certaine «superstructure névrotique individuelle" inconsciente. La forme est envisagée, en substance, comme le moule de la constitution névrotique du créateur. La conception psychologique de la forme n'est pourtant pas fondamentalement nouvelle. Elle part de l'idée traditionnelle que le style individuel reflète la constitution psychologique de l'artiste, et la forme se présente ici comme une configuration stylistique qui se reproduit et se répète en permanence. Eisenstein est sans aucune ambiguité. En outre, il parle du "système entier de formes comme l'état accompli [...] qui répond au concept de style selon l'emploi qu'en font les Allemands". Le lien de la forme et du style avec "la physionomie du réalisateur", comme il dit, montre jusqu'à quel degré le théoricien enracine la forme dans la psychologie individuelle du créateur. Dans «RG», Eisenstein souligne cependant qu'il s'agit précisément de la dynamique, et non pas du concept statique de style:

En géneral, le concept de "style" appliqué aux formes de l'art révolutionnaire est une grande question car le style est le produit d'une méthode canonisée, constituée et finie (un dogme) et la forme de l'art révolutionnaire répond au caractère changeant profond de la tactique (une dynamique).

Comme nous le verrons par la suite, la forme, dans « $R G$ », est effectivement le résultat d'un processus psychologique (de sublimation) et en ce sens, il s'oppose effectivement en apparence au 
concept de style statique ou de stylisation dont l'apparition chez Kouléchov est appelée par Eisenstein «un produit distillé de sublimation ", c'est-à-dire non pas le résultat d'un processus psychologique, mais la copie directe d'une forme déjà prête comme fétiche.

Sans aucun doute, l'usage actif de la méthode psychanalytique est le moment le plus intéressant de "RG». La psychanalyse utilisée ici massivement par Eisenstein, pour la première fois, offre de toute évidence une alternative à la réflexologie. Dans les textes plus anciens sur le montage des attractions, Eisenstein pense encore en termes de chaîne d'associations. Le principe de l'association d'idées, qui remonte à Locke, est central dans la philosophie du XVII' siècle: transformé par William James en principe d'association de stimuli, il est devenu la base de la réflexologie pavlovienne. Par la suite Eisenstein renoncera résolument à cette vision, pavlovienne dans son essence.

On sait qu'aux environs de 1928 - il le dit lui-même dans "Hors cadre" (1929) —, Eisenstein entre en polémique avec Poudovkine au sujet du montage "constructif", compris comme une accumulation de moments associatifs à la manière de la réflexologie. Il expose alors que l'impossibilité d'accumulation associative s'explique par le concept même de choc, de coup, de "moment agressif» (1976a, p. 40):

Lenchaînement n'est qu'une possibilité ou un cas particulier dans ma conception.

Rappelez-vous la quantité infinie de combinaisons que connaît la physique dans la question de la collision des sphères.

Selon qu'elles sont élastiques, non élastiques ou les deux à la fois.

Parmi ces combinaisons il en existe même une où la collision se dégrade jusqu'au mouvement uniformément accéléré des deux [sphères] dans la même direction.

Cela correspondrait au point de vue de Poudovkine.

L'accumulation d'associations en psychologie s'aligne sur le principe de l'habitude, encore employé par James, qui avait démontré que les associations et les processus psychiques qui leur 
sont liés actualisent les chemins frayés préalablement dans le cerveau. Ainsi, les associations se fondent toujours sur la répétition, l'usage réitéré de liens associatifs déjà constitués. En ce sens, elles sont plus proches du style statique. En fait, l'activité associative s'oppose à la création. La psychanalyse propose un autre tableau de transformation du traumatisme, plus complexe que la simple association. Il est évident que dans " $R G$ ", nous avons affaire à une première tentative de penser l'attraction (en tant que moment traumatisant) en dehors du paradigme de la réflexologie.

Les rapports d'Eisenstein avec la psychanalyse revêtent un formidable intérêt qui demeure à ce jour encore non élucidé . $^{\text {. }}$ Eisenstein découvre la psychanalyse au printemps 1918, à Gatchina. Le premier livre de Freud qui tombe entre ses mains, Un souvenir d'enfance de Léonard de Vinci, fut, selon lui, "une révélation inattendue de tout un domaine, où [il s]e précipite même sans guide" (1995, p. 354-355). Il est parfaitement évident qu'il découvre dans le Léonard de Freud ses propres traits. Cette coïncidence le frappe durablement et fait de ce livre qui est loin d'être le plus essentiel dans le corpus freudien presque le plus important pour lui. Petit à petit le freudisme est cependant refoulé dans ses écrits au profit d'une psychologie «désexualisée» de type ranko-jungien. Cette évolution se prête difficilement à l'explication. Une des raisons probables du rapport ambigu d'Eisenstein à Freud se trouve dans l'association de Freud à Meyerhold et à la figure du père (1978a, p. 66 et p. 152-159; 1980b, p. 20-26). Mais cela est loin d'en épuiser l'explication.

Dans «RG», parmi ses sources principales, outre Freud, Eisenstein nomme Otto Rank et Hans Sachs. Dans Mémoires, il déclare qu'après le livre sur Léonard, son initiation à la psychanalyse a passé par leur livre Die Bedeutung der Psychoanalyse für die Geisteswissenschaften [la signification de la psychanalyse pour les sciences de l'esprit] (1995, p. 355). Cet essai de 1913 va jouer dans la théorisation d'Eisenstein un rôle très important. C'est probablement lui qui suggère au cinéaste un modèle alternatif au travail des associations, différent de celui de la réflexologie. C'est encore ici que le principe de la formation de la forme 
se formule de manière nouvelle pour lui. L'origine de l'essai de Rank et Sachs se trouve dans l'affirmation de Freud selon laquelle le contenu de l'inconscient subit des changements radicaux dans le passage au conscient en raison des mécanismes de répression et de refoulement. Freud a montré que le travail du rêve se ramène à des déformations complexes du contenu de l'inconscient par déplacement, condensation, etc. Rank et Sachs décrivent tout un système de figuration employé lors de ce passage au conscient: l'inversion, la dissociation (découpage en morceaux), la représentation par son contraire, etc. Ainsi, le passage au conscient est-il accompagné d'une émergence de la forme qui peut se décrire précisément comme forme artistique, rhétorique, fondée sur un système complexe de tropes.

Rank et Sachs remarquent :

Le facteur formel auquel doit se soumettre l'inconscient, au moment de son introduction dans le conscient qui arrive parfois, est lié à la forme dramatique qui n'émerge pas moins dans ses manifestations culturellement signifiantes, en particulier artistiques, que dans la vie des rêves (1964, p. 19).

Pour eux, la manifestation suprême de la forme est le symbole, qui émerge des figures de discours et oscille de manière idéale entre l'expression d'un contenu caché et la présence de plusieurs couches du sens apparent. Ils envisagent le symbole comme une formation de compromis. En gros, la symbolisation se passe dans la sphère de l'inconscient, bien que le symbole appartienne au domaine du conscient, responsable de sa forme et de son interprétation. Puisque la forme (y compris artistique) émerge dans ce passage de l'inconscient au conscient, elle présuppose obligatoirement une base névrotique de la création, c'est-à-dire un contenu instinctif ayant subi la censure et le refoulement. Dans la mesure où Eisenstein accepte cette interprétation psychanalytique, pour lui nouvelle, de la forme et de l'association (qui tout d'abord lie la forme et le contenu caché symbolisé par elle ${ }^{5}$ ), il est contraint de tomber d'accord avec l'idée freudienne selon laquelle la névrose est la base de toute création. 
La question est de savoir quelle névrose précisément. Rank et Sachs sont, dans leur essai, sans ambiguïté à ce sujet. Pour eux, la création artistique émerge sur la base d'instincts sadiques et masochistes (et Eisenstein les suit complètement). Ils affirment que le plaisir procuré par l'œuvre d'art n'est pas séparable de la peur, de l'ennui, de l'horreur inspirés par son contenu. En un mot, le plaisir esthétique n'est pas séparable d'un sentiment pénible. Rank et Sachs croient que:

[...] l'objet esthérique existe dans un régime, proche du rêve diurne, où l'homme "joue" souvent les visions les plus pénibles pour lui. [...] Le plus souvent ce sont les visions de sa propre mort, ainsi que des tortures et les malheurs des autres, souvent sont représentées la misère, la maladie, la réclusion et la disgrâce $[\ldots]$; non moins souvent sont représentés l'accomplissement du crime monstrueux et sa découverte (1964, p. 101).

Selon eux, ce sont les tendances les plus agressives, c'est-à-dire les plus asociales qui procurent le plaisir maximal dans l'art, parce qu'elles subissent le refoulement le plus intense. Par conséquent, leur irruption dans le conscient est d'un point de vue énergétique la plus puissante, bien qu'elle soit accompagnée de toutes sortes de déformations et d'altérations qui rendent ces tendances socialement acceptables. Comme nous le savons déjà, c'est en ces déformations que consiste l'émergence de la forme.

La deuxième raison importante pour laquelle ce sont les instincts sadiques qui se trouvent à la base de la création artistique tient à leur origine infantile. Comme l'écrivent Rank et Sachs, ces tendances trouvent, après un refoulement profond à l'âge adulte, un refuge dans les rêves diurnes, d'où ils arrivent dans l'œuvre d'art. Mais la partie probablement la plus intéressante de l'essai de Rank et Sachs concerne l' «économie " de la forme du texte artistique. Il s'agit ici d'une

[...] construction qui ne laisse pas vainement l'affect brû-
ler toute son énergie d'un seul coup, mais qui le fasse s'é-
lever lentement et graduellement d'une étape à l'autre,
jusqu’à ce que le plus haut degré soir atteint et que l'affect
soit alors abréagi sans réserve. La "forme artistique in-
terne" qui contraint l'artiste à choisir pour chaque

La sublimation comme formation de la forme. Note sur un article inédit d'Eisenstein 
matière une forme propre de traitement, n'est rien d'autre que la compréhension inconsciente du fait que le maximum de quantité d'affect que puisse produire l'objet pourrait être atteint par le balancement le mieux calculé entre la progression et le retardement (1964, p. 105).

Cette économie de la forme mène à un profit énergétique colossal de la perception de l'œuvre d'art par rapport à la réalité et par conséquent, à un profit sensible dans le plaisir. Rank et Sachs utilisent ici le modèle de la "réaction", élaboré par Freud dans son livre sur le mot d'esprit. Chez eux le concept de forme est profondément psychologisé, et il est très proche de ce qu'Eisenstein évoquait dans "La Méthode de réalisation du film ouvrier", où la forme était envisagée comme la réalisation d'un enchainement de chocs et de bouleversements dans le matériau. Ici, il s'agit également des affects dus aux chocs et de la profondeur "attractionniste" maximale de l'impact. L'ébauche psychologique de la forme était alors décrite en substance comme la combinaison de la "figuration" déformante et de la "structure" économique complexe fondée sur le retard et le mouvement. Il est important, bien sûr, de savoir qu'à l'époque, Eisenstein travaillait dans ses articles sur la problématique du mouvement expressif qui, selon lui, se fonde sur l'interaction de la motricité et du retard, de l'inhibition.

Le processus décrit par Rank et Sachs avait été auparavant identifié par Freud comme celui de la "sublimation". Freud évoque souvent la sublimation, mais il n'a finalement pas donné d'étude détaillée de ce phénomène. On peut dire qu'elle est une réorientation de la libido des objectifs sexuels vers d'autres objectifs. Elle surgit encore à l'époque de la sexualité enfantine, quand l'enfant n'a pas la possibilité de réaliser ses pulsions sexuelles. Comme l'indique Freud dans ses Trois Essais sur la sexualité, dans le processus de la sublimation, les excitations sexuelles «font entrer en jeu des contre-forces, ou des réactions" (des pulsions réactionnelles) d'où surgissent les sentiments d'aversion, de honte et de morale (1962, p. 71). La sublimation fait partie des formations réactionnelles (Reaktionsbildung) et, en tant que telle, avoisine les trois autres espèces de transformation réactionnelle des pulsions: le "retournement en son contraire", l' «inversion du rapport à l'objet " et la «répression" (Freud, 1963b, p. 91). En ce 
sens, la sublimation est effectivement proche du sado-masochisme qui inverse l'amour en son contraire - la haine - , ou du narcissisme qui inverse le rapport à l'objet de la pulsion en tournant la libido sur soi ${ }^{6}$. La sublimation a affaire aux mêmes paires d'oppositions instables que les instincts quand ils connaissent la répression: "Sujet (ego) — Objet (le monde extérieur)"; "Plaisir — Douleur"; "Actif - Passif"" (Freud, 1963b, p. 97).

La sublimation est un des thèmes centraux du livre sur Léonard. Freud y étudie la façon dont elle engendre le génie de Léonard en apparaissant en réaction au sexuel (il cite les mots mêmes de Léonard selon lesquels «l'acte de l'accouplement et les membres qui y sont employés ont une laideur telle [...]" [Freud, 1973, p. 21]) et il montre que la passion du peintre pour ses recherches relève d'une curiosité sexuelle infantile sublimée qui n’a pas été orientée vers la vie sexuelle ou a été réprimée, et qui a été «transformée " en son contraire et désexualisée.

Les idées de Freud ont exercé une telle influence sur Eisenstein qu'il envisageait en grande partie ses propres problèmes liés à la sexualité à travers le prisme de la sublimation. Il y rapportait, non sans raison, «l'inhibition devant le but», courante chez lui. Par exemple, il écrit à Péra Attachéva qu' "il n'avai $[t]$ jamais été jusqu'au bout avec les objets de ses relations amoureuses ${ }^{7}$ " (1997-1998, p. 234) et généralisait:

Je me suis plaint auprès de vous à plusieurs reprises d' "inhibitions" dans toutes mes manifestations. Une sur-inhibition.

Il va jusqu'aux plus petits détails :

[...] il me suffit de vouloir quelque chose (un rien du tout) pour qu'instantanément je fasse tout pour ne pas l'obtenir. Je considère cela depuis longtemps déjà, comme un transfert de la sphère sexuelle où la superascèse a développé à tel point les forces de répression et leur activation automatique immédiate - (il suffit pour le désir d'apparaître) - que cela se répand sur toute activité en général (1997/1998, p. 234-235)!

La sublimation est si importante pour l'activité d'Eisenstein que lorsqu'il souffre de problèmes avec la mise en forme de ses projets, 
c'est-à-dire avec la sublimation, il consulte des psychanalystes. Il sera alors hanté par la peur que l'analyste ne le prive de la base névrotique de sa création hors de laquelle il ne pouvait penser la production artistique. Ainsi en 1930, au sujet du traitement d'un psychanalyste, il écrit de New York, à la même Péra Attachéva, que "celui-ci a décidé de détruire à la racine ce qui gêne ma névrose (ne pas toucher aux autres)» (1997/1998, p. 229), or la préservation de sa névrose est pour lui la chose la plus importante...

Il semble qu'Eisenstein se représentait la psychanalyse en tant que doctrine à travers le prisme de la sublimation, c'est-à-dire de la désexualisation. En tout cas, dans les années quarante, il formule une évaluation générale de la psychanalyse comme doctrine «transitoire" $(1997$, vol. 2, p. 79):

Je l'ai toujours représentée [l'école freudienne] comme quelque chose de "transitoire" - comme un "stade transitoire" sur la voie de l'acquisition de bases beaucoup plus larges et plus profondes pour lesquelles le secteur coloré par le sexe n'est qu'un domaine particulier.

Dans «RG» Eisenstein compare sa propre névrose, productive pour la création, à deux névroses "non productives", celles de Kouléchov et de Room. Selon lui, Kouléchov est fétichiste. Le fétichisme est l'inversion d'une pulsion sexuelle, apparentée à la sublimation. Voilà ce qu'il écrit de ses films, dans " $\mathrm{RG}^{8}$ " :

[...] je ne peux pas ne pas relever le matériau des plus "glissants" du fétichisme primitif - la prééminence du pied et de la chaussure pointue - sous le signe duquel se tournent Rayon et West. Sans parler des jambes inoubliables de Khokhlova - "vis motrix" de ces choses $[\ldots]^{9}$.

Le fétichisme a été abordé par Freud dans son Léonard. Il l'expliquait par le désir passionné du garçon de voir le sexe de sa mère, qu'il se représente tout d'abord comme pénis. La découverte que la mère n'a pas de pénis produit un traumatisme, une profonde angoisse de castration et, force du mécanisme réactif, lie le sentiment de dégoût à l'image du sexe féminin. Le fétiche supplée le pénis absent de la mère: 
Le fétichisme du pied et de la chaussure féminins ne semble prendre le pied que comme un symbole d' "ersatz" du membre de la femme, adoré du temps de l'enfance, depuis lors regretté (Freud, 1973, p. 73-74).

La proximité du fétichisme et de la sublimation tient à ce que le fétiche est un «ersatz» acceptable et très accessible du génital. Cependant le fétichisme n'est pas acceptable pour Eisenstein pour deux raisons complémentaires. Premièrement, il exprime "l'idéologie de la passivité" — dans le système d'oppositions freudien, il renverse l'" actif" en "passif". Eisenstein ne s'attarde pas par hasard de façon si détaillée sur l'opposition entre le fétichisme des chaussures et des pieds chez Kouléchov et le caractère actif des mouvements de ces mêmes pieds. En raison de sa passivité, le fétichisme n'est pas un miroir du processus psychologique, il se donne dans une forme déjà prête, dans la forme du fétiche statique, du style, du "produit distillé de sublimation". Cette passivité est liée à un autre trait relevé par Freud. Le fétichisme délivre l'homme "de la peur horrifiante de la menace de castration à la vue du sexe féminin» (1963b, p. 216). Il est antitraumatique, et par conséquent, antiattractionniste, si l'on considère que l'attraction est fondée sur le choc. En fin de compte, Eisenstein rejette le "style» kouléchovien en tant qu'idéaliste et passif, incapable d' "activer" le spectateur.

Le deuxième exemple - Course à la gnôle de Room - est le contraire du fétichisme kouléchovien. Si Le Rayon de la mort est un exemple de forme déjà prête, passive, Course à la gnôle est, au contraire, l'exemple du matériau brut, incapable d'acquérir une forme. Il est curieux que l'exposition de ce matériau primitif non mis en forme soit rattachée par Eisenstein à la réflexologie: le film de Room est décrit en termes de "technologie de la formation des réflexes conditionnés - tout selon Pavlov". Voici comment, dans «RG», Eisenstein définit l'essence de la création de Room :

Room scuffre d'une névrose - je ne sais pas précisément comment elle est désignée par la science - de jouissance du dégoûtant, lié en grande partie au processus de prise et de rejet de la nourriture (la nourriture «rebutante", les vomissements, le fait de se souiller 
avec de la nourriture, etc.) et à la laideur due aux transgressions physiques des normes, en particulier dans le domaine de la "structure matérielle" (de la peau et autres tissus - les teignes, les difformités, les maladies de peau et, enfin, les haillons déchirés couverts de poux... ${ }^{10}$ ).

La sensation de dégoûtant est liée à celle de chaotique... Le dégoûtant renvoie à la sphère de l'impact attractionniste des chocs, et Eisenstein souligne que l'attractionnisme se manifeste pleinement ici et possède une base réflexologique articulée. Pourtant la "méthode» de Room n'est pas acceptable pour Eisenstein, précisément en raison de son absence de forme, ou, comme il le dit dans l'article, de son "défaut de sublimation". En effet, ce qu'Eisenstein décrit, c'est une forme infantile de manifestation de la sexualité, liée à l'acte de manger l'objet de la pulsion, "au type d'amour qui se combine avec l'anéantissement de toute existence séparée de l'objet" (Freud, 1963b, p. 102). Un sadisme infantile non surmonté. Le non-dépassement du stade infantile consiste précisément en l'absence de forme, dans la nature infecte des vomissements et des teignes qui se dispersent, qu'Eisenstein décrit. Le dégoût est une étape importante dans le devenir du fétichiste. Dégoût du sexe féminin dont la vue rappelle la menace de castration. Chez le fétichiste, le dégoût, lié à l'absence de forme, est cependant vite dépassé par la forme figée du fétiche - par l'éclat épidermique du bout étroit du soulier. Chez Room, le dégoûtant est préservé et ne subit pas de mise en forme, c'est-à-dire de sublimation:

Room, séduit par la proximité et l'abondance du matériau collecté soi-disant pour des motifs utilitaires, et qui l'agite, ne l'a pas soumis au processus de sublimation et à la tâche posée devant soi, mais, au contraire, il a succombé à cette tentation et est allé par une autre voie, la ligne de la satisfaction de la névrose dans les formes du film qu'il était en train de créer, et non du dépassement du principe névrotique.

Et c'est là toute la tragédie ${ }^{11}$.

Aux yeux d'Eisenstein, Kouléchov et Room expriment deux types de réalisations inacceptables. Il leur en oppose un troisième auquel il rattache Griffith, Stroheim et... lui-même : 
La puissance et la force captivante de leurs œuvres a lieu, certes, au prix d'énormes défauts et déformations dans leur base originelle nerveuse.

La forme caractéristique du sadisme sublimé marque le côté formel de leurs œuvres, en les rendant incomparables par leur effet bouleversant.

La cruauté et l'absence de pitié - le sadisme, la névrose fondamentale, nécessaires pour le réalisateur en général - - le réalisateur se retrouve parmi les produits de sublimation du sadisme du côté du chirurgien et du... boucher ${ }^{12}$.

Cette déclaration est l'une des plus choquantes de l'Eisensteinthéoricien. On peut largement l'expliquer par Rank et Sachs et le privilège quaccordent ces auteurs au sadisme, ainsi que par la théorie de l'attractionnisme qui découle de l'attitude sadique. Et pourtant il n'est pas facile de comprendre ce qui se passe exactement avec les pulsions sadiques et de quelle façon elles sont transgressées et sublimées en forme. Le sadisme à son stade primitif, infantile, mène au domaine du sans-forme. Il est, à proprement parler, dirigé contre la forme en tant que principe destructeur actif. La sublimation est, dans ce contexte, le "salut» de la forme, puisqu'elle fait manquer son but originel au sadisme - la destruction. Freud remarque, dans une lettre à Marie Bonaparte (27 mai 1937), que tout déplacement, toute déformation de l'intention originelle est également destructrice, sadique dans son essence. C'est pourquoi le sadisme agit simultanément contre la forme, en la détruisant, et au nom de la forme, en la créant par la déformation de la pulsion originelle destructrice:

Toute activité qui restructure ou apporte des changements est à un certain degré destructive, et fait ainsi manquer son but destructeur originel à une partie de l'instinct. Nous savons que même l'instinct sexuel ne peut pas agir sans une part d'agressivité. Ainsi, dans la simple combinaison de deux instincts il y a déjà une sublimation partielle de l'instirict destructeur (Jones, 1957, vol. III, p. 464).

Mais la conception la plus développée de la sublimation comme transgression des composantes sadiques se trouve proba- 
blement dans les travaux de Melanie Klein dont les conclusions correspondent, de manière étonnante, au caractère de la méthode eisensteinienne. Selon elle le sadisme, orienté vers les objets, force l'enfant à attendre une réaction en réponse à son action sadique de la part de ses objets, et, par conséquent, engendre un sentiment de peur et d'inquiétude :

\begin{abstract}
Puisque l'enfant veut détruire les organes (le pénis, le vagin, le sein) qui remplacent les objets, il commence à avoir peur d'eux. Cetre peur contribue à l'établissement d'équivalences entre les organes dénommés et les autres choses; sur la base de ces équivalences ces choses deviennent aussi les objets de la peur, et ainsi l'enfant est obligé de créer de nouvelles équivalences qui sont la base de l'intérêt pour les nouveaux objets et la source du symbolisme (1984, p. 220).
\end{abstract}

Ainsi, la destruction de la forme permet à un système d'équivalences de se constituer et d'apparaître à la conscience symbolique, qui se sert de signes - cette conscience est le produit principal de la sublimation. Le mode de penser d'Eisenstein, avec sa curiosité infatigable et l'enchaînement infini d'analogies et de substitutions qui croît à partir de son sadisme originel, est une illustration parfaite du modèle kleinien de la sublimation ${ }^{13}$.

Comment donc la sublimation du sadisme en une forme s'effectue-t-elle concrètement chez Eisenstein? Il est vain de chercher la réponse à cette question dans " $R G$ " ou dans quelque autre texte. Proposons donc au lecteur une solution hypothétique sur la base de textes ultérieurs du réalisateur.

On a vu que le réalisateur "se trouv[ait] du côté du... boucher». Rappelons que dans "Le montage des attractions au cinéma ", il donnait deux exemples d'organisation de montage, tous deux rattachés à ce côté «boucher». Dans le premier:

[...] le cou est empoigné, les yeux montent vers le front, le couteau est levé, la victime ferme les yeux, sur le mur gicle le sang, sur le plancher tombe la victime, la main essuie le couteau [...] (1974, p. 130).

Plus tard, dans ses "Mémoires", Eisenstein a essayé de reconstituer la généalogie de cette imagerie sadique en la faisant 
remonter à des images obsédantes de boucher qui hantèrent son enfance :

Je fixais mon regard sur un point et je voyais devant moi les bouchers ivres, $[\ldots]$ le corps suspendu et le crochet horrible.

[...] Je ne "voyais" alors jamais de sang. Les lambeaux de peau et de corps étaient arrachés, comme de la cire, laissant des bandes sanglantes, sans baigner dans le sang (1995, p. 532).

L'absence de sang est ici remarquable. Elle oppose à l'évidence la fantaisie d'Eisenstein au liquide, au muqueux, au sans-forme roomiens. Tout se passe comme si le sadisme d'Eisenstein, dès l'origine, maniait des formes. Ce fantasme infantile va se répéter sous une autre forme, sublimée, dans certains films du cinéaste. Dans un cas, il prend une apparence qui serait difficilement identifiable si Eisenstein ne la désignait lui-même. Il s'agit de l'épisode de la transformation du deuil de Vakoulintchouk en révolte populaire dans la troisième partie de Potemkine. Il y a là un plan très bref d'un jeune homme qui déchire sa chemise. Cet acte est l'une des reprises de la lacération de la peau dans le fantasme infantile qu'on vient d'évoquer. La chemise déchirée apparaîtra encore dans Octobre et Eisenstein le reliera directement à l'épisode correspondant de Potemkine (1995, p. 563). Voici comment il est décrit dans les "Mémoires":

Ce fragment minuscule n'est probablement même pas perçu comme "objet", mais seulement comme accent dynamique - un paraphe univoque sur le cadre, sans qu'on ait le temps de voir ce qui s'y passe réellement.

Or ce qui s'y passe c'est cela :

c'est exactement dans ce fragment qu'un jeune homme, au paroxysme de sa colère, déchire sa chemise.

Ce fragment-là, comme point culminant, est nécessairement situé entre l'étudiant en colère et les poings qui sont en train de s'envoler, se sont déjà envolés et sont brandis en l'air (1995, p. 525).

Eisenstein explique la nécessité de cet accent dynamique par le fait que, pour créer un effet, il faut obligatoirement mettre un "morceau accentué" entre l'"accumulation" et l' explosion", 
qui "dessine l'explosion" de manière précise (1995, p. 525). Cette explosion se présente ici comme une plaie symbolique, une rupture de surface sans profondeur. La curieuse propriété de ce fragment accentué tient au fait qu'Eisenstein en souligne ce caractère de surface. La chemise du jeune homme est, dans ce cas, l'équivalent direct de la peau derrière laquelle le sang ne coule pas et qui se détache par bandes, comme de la cire. Bien sûr il n'est point fortuit qu'Eisenstein appelle ce plan "paraphe sur le cadre", "acte de dessiner-à-travers". Il s'agit précisément d'un paraphe métaphorique sur la surface, d'une ligne, d'une arabesque, d'un contour, d'une sorte d'écriture. Bizarrement la violence sadique sur le corps transforme le corps en une surface, un paraphe couvert de monogrammes. Il arrive quelque chose de semblable aux figures déformées des moines sur les tableaux de Magnasco:

[...] ils semblent être des monogrammes tordus et extravagants, assis dans des habits de moines, chauffant auprès du feu leurs queues, boucles, nœuds, articulations disjointes (1995, p. 304).

Cette écriture sadique de surface est probablement liée à l'inversion de l'instinct décrite par Freud - du sadisme en masochisme, du voyeurisme en exhibitionnisme - , qui est, en fin de compte, fondée sur l'instabilité des rapports entre le dehors (l'objet) et le dedans (le sujet ${ }^{14}$ ). Eisenstein se rendait parfaitement compte de cette ambivalence dans les rapports du sujet et de l'objet dans le sado-masochisme:

[...] la limite entre le sujet et l'objer de cruauté s'efface.

Il n'y a pas de "distribution " précise de tôles.

$\mathrm{Et}$, en s'intéressant à la sensation de douleur, on est obligé de s'infliger la douleur soi-même (1995, p. 541).

La dissolution des différences entre le sujet et l'objet passe par le paraphe, la ligne, l'arabesque où disparaît la différence entre le dehors et le dedans, le contour et le corps du volume, entre l'écriture et le mouvement de la main. L'épisode de la lacération de la chemise répète à sa manière un épisode d'un film, probablement produit par Pathé, que le réalisateur a vu dans son enfance et qui est resté présent à sa mémoire: 
La jeune femme d'un forgeron trompe son mari avec un jeune sergent de l'Empire.

Le mari l'apprend.

Il attrape le sergent.

Le sergent est attaché.

Il est jeté dans le fenil.

Le forgeron déchire son uniforme.

Il dénude son épaule.

Et... lui marque l'épaule au fer rouge.

Je m'en souviens comme si c'était hier: l'épaule nue, l'énorme barre de fer dans les bras musclés du forgeron aux favoris noirs, et la fumée (ou la vapeur) blanche qui s'élève de l'endroir de la brûlure ${ }^{15}$ (1978a, p. 170).

Le choc de cette scène est également lié, selon toute apparence, au côté inattendu de la chose. La lacération de l'uniforme, la barre de fer pointée sur le corps, produisent quelque chose de bizarre - pas une plaie, pas une déchirure de la peau, mais une marque - , une écriture, un texte, le signe de sublimation "par excellence». L'écriture ne pénètre pas l'intérieur du corps, au contraire, c'est comme si elle en émanait avec la fumée blanche. Le mouvement vers le dedans se transforme en mouvement vers le dehors. Dans la conscience d'Eisenstein cette scène de film ancien s'est fixée comme quelque chose d'extrêmement ambivalent et radicalement destructeur des rapports sujet-objet:

Mais cette scène de marquage demeure indélébile dans ma mémoire jusqu'à maintenant.

Dans mon enfance, elle me torturait de cauchemars.

Elle se représentait à moi la nuit.

Tantôt je me voyais en sergent.

Tantôt en forgeron.

Je saisissais ma propre épaule.

Parfois elle me paraissait être la mienne.

Parfois celle d'un autre.

Il devint difficile de savoir qui marquait qui ${ }^{16}$ (1978a, p. 171).

Le transfert de la torture dans la sphère de l'écriture, l'équivalence entre la peau et le papier sont, bien sûr, des moments de sublimation qui dépassent la cruauté, la transforment en une 
sorte de "création". Melanie Klein (1960, p. 68) décrivait une petite patiente sadique qui s'était mise tout d'un coup à découper du papier pour en faire des ornements:

Pendant qu'elle faisait cela, elle m'a dit qu'elle préparait une "farce" et que le sang coulair du papier, explique M. Klein. Le découpage de papier obéissait à des motivations très diverses. Il offrait un exutoire aux pulsions sadiques et cannibales, mais en même temps servait des tendances réactionnelles puisqu'il exprimait aussi un principe créatif. Les beaux ornements découpés représentant une nappe, par exemple, signifiaient les parties génitales de ses parents ou le corps de sa mère, rétablis de la destruction que sa fantaisie leur avait fait subir ${ }^{17}$.

Ce qui se passe au moment de la transformation du paraphe en lacération et de la lacération en paraphe, ce moment d'échange instable, peut être compris comme un processus de sublimation. Il s'agit de transformer un geste sadique en quelque chose d'autre, mais cet "autre", ne possédant pas de forme statique, ne devient pas un fétiche "naissant" de la plaie comme signe de l'absence, de la castration. C'est le processus dynamique instable qui occupe la place de la forme statique et c'est lui qui, en fin de compte, devient le processus de formation du sens. Eisenstein souligne qu'un des impératifs essentiels pour produire la sublimation de la forme, c'est la vitesse de déroulement du processus, l'impossibilité de sa stabilisation:

L'introduction et l'expulsion de l'ordre du vécu infantile par les associations liées à la zone "orale" sont si rapides et si instantanées qu'elles n'ont pas le temps de "se transformer en marais" dans le limon de la "stagnation " psychologique et ne permettent pas la perception de schwelgen, comme les Allemands désignent très bien le complexe de sensations émotionnelles de toutes sortes où apparaît tout acte psychologique de gourmandise qui procure le plaisir (1995, p. 556).

D'où l'équivalence entre le paraphe-déchirement et l'explosion et l'insistance à souligner de mille façons le dynamisme maximal et la richesse conflictuelle de la forme en devenir. Cette composante explosive est tout à fait repérable dans l'intérêt 
d'Eisenstein pour la graphologie, à laquelle renvoie l'équivalence entre déchirement de la surface et paraphe. Le moment du déchirement représente l'apparition de l'intervalle comme expression la plus dramatique du conflit des forces et, par conséquent, pour Eisenstein, le moment suprême de l'expressivité. C'est ainsi qu'à travers l'image de l'écriture peut apparaître l'image plastique ou l'image en volume d'hommes ou d'objets ${ }^{18}$. Eisenstein parle (1978b, p. 254) du passage de l'«imitation graphique" à l'«imitation plastique». Dans le processus de captation de l'image graphique, le linéarisme s'élargit en volume. Mais cette apparition de la profondeur (en particulier, dans l'intuition graphologique) se déroule à une vitesse limite, exprime un certain saut de la surface, de la ligne et en profondeur. Dans le cas de l'ouvrier qui déchire sa chemise, il n'y a pas de pénétration visible et, néanmoins, une certaine profondeur intelligible apparaît (la «lacération" se transforme en "explosion"). C'est précisément cette profondeur invisible qui intéresse le réalisateur. Un semblable processus est défini par Melanie Klein (1984, p. 220) comme "processus de symbolisation", lié par elle au sadisme infantile 19 .

Dans les années trente, Eisenstein liera un "saut" de ce type au pathos et à l'extase comme formes physiques de dépassement et de passage à une qualité nouvelle. Dans La Non Indifférente Nature, il s'attarde sur la déchirure fictive de la surface de l'écran par la proue du "Potemkine" qui fonce vers le spectateur à la fin du film et il explique l'origine de cette "déchirure»:

Dans l'idée de la réalisation, le dernier cadre du film la proue du cuirassé - devait fendre... la surface de l'écran : l'écran devait se déchirer en deux [...].

Par ce procédé Potemkine parachevait une série de cas analogues dans le passé.

Une fois, comme Kazimir Malévitch me l'a raconté plus tard, le rideau s'était déchiré de cette façon en ouvrant la première représentation des «boudetlianie" les futuristes russes - au théâtre de la rue Offitzerskaïa $[\ldots]$.

C'est dans une tout autre montée de pathos social, que se déchirait à une tout autre date - le mémorable 14 juillet 1879 - le rideau de gaze qui séparait les 
spectateurs des acteurs dans le petit théâtre parisien Des délaissements comiques* $[\ldots]$.

Le 14 juillet, la nouvelle de la prise de la Bastille parvient à Plancher-Valcour et, au paroxysme d'un pathos véritable, il crève de son poing le rideau de tulle, le déchire en deux [...].

Et, enfin, nous pouvons rapporter ici le cas le plus connu dans la littérature d'une explosion pathétique accompagnée d'une déchirure de rideau, celui du Temple au moment de la tragédie du Golgotha (1976b, p. 92-94).

En principe, les exemples énumérés semblent non pas illustrer l'idée de l'apparition de l'abstraction (si on conçoit la profondeur comme abstraction) par l' "explosion", par le "saut», mais comme régression de la convention (la surface de l'écran) à une matérialité agressive (dans la déchirure du rideau apparaît un poing parfaitement réel). Mais cette matérialité n'est autre que le résultat de l'inversion du rapport entre sujet et objet. Tout se passe comme si la marque du forgeron du vieux film Pathé sortait de la surface de l'écran et frappait l'épaule du spectateur (Eisenstein lui-même). Dans son étude "Sur le cinéma en relief», Eisenstein parlera du "principe de l“"irruption" des éléments du spectacle au cœur de la salle" (1986a, p. 108-118, 128-141).

L'inversion n'est ici que la condition primaire, derrière laquelle apparaît le produit dynamique de la sublimation de la forme. Ce qui est essentiel ici, ce n'est pas tant l'irruption directe dans la salle, par exemple les canons pointés du «Potemkine", que la déchirure du rideau lui-même, la formation de rupture ou le dédoublement du graphe qui lui correspond. Ce dédoublement de la ligne est, en même temps, sa désagrégation entre le monde du visible et celui de l'invisible auquel elle donne l'accès. Cet accès apparaît sur la base de l'inversion du sujet et de l'objet. Si on découpe la surface en suivant le paraphe, le paraphe éclatera et apparaîtront les deux côtés de la plaie qui ouvre sur l'intérieur. Quoique chez Eisenstein, derrière la chemise déchirée, s'ouvre non la profondeur mais la surface d'un autre tissu, la peau, l'effet est le même. Le dédoublement de la ligne par la déchirure crée un effet de non-coïncidence des contours, auquel Eisenstein attachait une signification particulière. 
C'est à cela qu'il liait, on le sait, ledit "Ur-phänomen» du cinéma, c'est-à-dire l'illusion primaire du mouvement, conçue comme idée de mouvement. Curieusement dans "Stuttgart" (1929), il interprète la non-coïncidence des contours entre les photogrammes (l'intervalle) en termes de stéréoscopie, c'est-àdire de profondeur en devenir. L'idée de profondeur apparaît ainsi comme résultant de la non-coöncidence des contours, et de la même façon le mouvement se donne à nous, au cinéma, comme une certaine abstraction, engendrée par l'intervalle entre les contours, leur non-coïncidence :

[...] de même naît aussi d'autre part le phénomène de la profondeur spatiale en tant que superposition optique de deux surfaces dans le cas de la stéréoscopie.

De la superposition de deux grandeurs de même mesure nait en somme une nouvelle dimension plus élevée.

Ainsi se produit ici, dans le cas de la stéréoscopie, la superposition de deux dimensionalités non identiques -. le phénomène de la tridimensionalité stéréoscopique ${ }^{20}$.

Ainsi, bien que la profondeur ne soit pas ouverte physiquement par la déchirure, elle est créée symboliquement par un phénomène de pure surface.

Eisenstein retourne au même thème en 1933 dans "L'erreur de Georges Méliès ". Après le récit selon lequel Méliès aurait filmé deux fois par erreur le même objet sur la même pellicule, et, par là, créé involontairement un effet de non-coïncidence des contours, Eisenstein déclare que c'est ce dernier phénomène qui est responsable de la perception de la profondeur de l'espace. Il est évident que l'"erreur" de Méliès n'a rien en commun avec l'effet de stéréoscopie réelle, mais Eisenstein affirme, contre toute évidence, que, dans ce cas, c'est l'intervalle qui est la base de la vision stéréoscopique parce que l'intervalle engendre l'abstraction, et il conçoit la stéréoscopie exactement comme dimension abstraite de l'idée. Il explique la signification du "saut", du passage de la non-coöncidence des contours à l'idée de profondeur en termes de passage à la représentation :

Les termes bidimensionnels - im-pression*, Ein-druck*, Im-press - à la différence des termes tridimensionnels- 
spatiaux - re-présentation, Vor-stellung* (1988, p. 259).

Cette affirmation n'est autre que le développement de la loi "dialectique " qui était formulée dans "Stuttgart » :

Sehr wichtig*.

L'activité de la pensée - activité corrélative - processuelle*...

(à la différence du Daseins-Prinzip* — statique de la perception).

Selon la loi de multiplication des dimensions (une projection en deux dimensions sur une autre projection en deux dimensions donne le concept d'un corps en trois dimensions), le processus de la pensée s'organise comme suit :

à travers la sensation, l'impression (im-pression*)

impression A

impression B

représentation $\mathrm{M}$

représentation $M$

représentation $\mathrm{N}$

concept $\mathrm{X}$

L'impression, c'est "presser" (press*, Druck*) l'équivalent de deux dimensions.

La représentation, c'est Vor-stellung* - l'équivalent de trois dimensions.

Et enfin, le concept, c'est prendre - "yat* " (Griff*)

- action active - l'équivalent de quatre dimensions

(on inclut la sensation temporelle, l'action active) ${ }^{21}$.

Le processus qu'Eisenstein décrit ici n'est autre que le processus de la formation dynamique de la forme comme résultat de la sublimation. Au début nous avons une "impression", une empreinte, une insertion, un geste sadique semblable au choc attractionniste. Cependant, l'impression laisse petit à petit la place à une "représentation" qui est déjà une "forme" sublimée, purifiée de la composante sadique, intelligible et tridimensionnelle. La profondeur, découverte par le découpage du boucher, est, en fin de compte, transformée en concept, c'est-à-dire que s'effectue exactement ce dont rêve Eisenstein - le transfert du matériau des réflexes au domaine de la création consciente, des idées. 
La sensation est, pour Eisenstein, l'équivalent de l'impression bidimensionnelle, de l'" empreinte", du signe sur le papier, sur la peau, du graphe, de la marque. Elle se trouve sur le plan de la déchirure primaire de la surface. La divergence de deux graphes, de deux "paraphes" crée l'énergie, nécessaire pour la déchirure de la surface et la découverte de la profondeur. Eisenstein souligne l'énergie de la pénétration, de la percée - "press*", "Druck*". L'intervalle, qui apparaît en raison de la noncoöncidence de deux empreintes, découvre aussi la dimension temporelle, en détruisant le statisme du Daseins-Prinzip.

Ce moment de déchirure de la surface a une signification fondamentale pour la structure du sens dans le film. C'est le moment du passage de la bidimensionnalité à la tridimensionnalité. En même temps sur la surface, la ligne subit, elle aussi, un changement qualitatif, elle découvre la profondeur et se dédouble. Le sadisme primitif subit une symbolisation sublimante, les signes émanent littéralement de lui. Le découpage des tissus à la boucherie se trouve, bien sûr, chez Eisenstein en rapport direct avec la manière dont il décrit «le matériau roomien, les défauts de la peau et autres tissus ou les teignes, les difformités, les maladies de peau ". Dans les deux cas, il s'agit d'une mutilation de la peau. Les tumeurs dégoûtantes chez Room n'ont nulle tendance à la sublimation, tandis que le paraphe-déchirure chez Eisenstein s'introduit instantanément dans la dynamique de la formation de la forme.

Sous ce rapport, il est opportun de rappeler la différence entre le signe et la marque, établie par Walter Benjamin dans son article "Peinture, signe ou marque" (1996, p. 84) :
[...] le signe est imprimé sur quelque chose, tandis que la marque y apparaît. D'où il est clair que le domaine de la marque, c'est le média lui-même. Tandis que le signe absolu n'apparaît pas dans la plupart des cas sur les êtres vivants, mais peut être imprimé ou peut appa- raître sur les immeubles sans vie, sur les arbres, etc., la marque apparaît en principe sur les êtres vivants (les stigmates du Christ, la rougeur, probablement, la lèpre et les grains de beauté).

Benjamin affirme que la peinture travaille principalement avec des marques, puisque les taches de couleur sur la toile 
appartiennent totalement au domaine du média, c'est-à-dire à l'illusion, et non à la matière physique de la toile. La marque émane de la sublimation du matériau, comme le transfert de l'immédiatement donné (par exemple, du dégoûtant) dans le domaine du symbolique. Il est significatif que Benjamin donne comme exemple de la marque des mutilations de la peau, semblables à celles dont Eisenstein parle au sujet de Room, - par exemple, la "lèpre". En même temps, la différence entre la marque benjaminienne et les mutilations roomiennes est considérable. La lèpre chez Benjamin se sépare instantanément du corps et ouvre la dimension symbolique (comme, par exemple, les stigmates), tandis que chez Room les "teignes" restent teignes jusqu’à la fin.

Ce qu'Eisenstein décrit comme déchirure, plaie symbolique, c'est le signe (le graphe, le contour), qui se transforme en marque. Et cette transformation est sublimation du matériau (par exemple, du traumatisme de la castration lié à la plaie) dans la forme artistique. Ce qui était déchirure - et ensuite "impression", "empreinte" — acquiert la tridimensionalité — et, en même temps, la signification métaphysique — et est transformé en marque du péché, de sainteté, de culpabilité, etc. L'épisode de la lacération de la chemise s'inscrit dans la problématique de la culpabilité, du péché, de la sainteté, associée à la ligne de Vakoulintchouk dans le film.

La marque se sépare de la surface et acquiert la tridimensionalité : Eisenstein lui-même souligne cette transformation quand il oppose l' «im-pression" - le mouvement dans la profondeur à la "Vor-stellung*" — la position devant la surface —, à la séparation d'avec elle. C'est le passage de la surface à la stéréoscopie symbolique, du signe à la marque, du paraphe au sens qui est, en fin de compte, possible grâce à la sublimation de la pulsion sado-masochiste primitive.

Traduction du russe: Egor Ovtcharenko

1. Gonka za samongonkoi, A. Room; Lutch smerti, L. Kouléchov. Seul le film de Kouléchov est conservé [NdÉ]. 
2. Le Bazar de la concupiscence est le script d'une tragicomédie satirique retrouvé dans les papiers d'Eisenstein après sa mort. Il est situé dans un bordel du front durant la Première Guerre mondiale. Eisenstein y fait brièvement allusion dans les "Mémoires " [NdÉ].

3. On ne peut exclure que le choix même de la grève comme sujet ait été dans une certaine mesure défriri par Les Méditations sur la violence (1908) de Georges Sorel qui dit que la grève générale est la quintessence de la violence révolutionnaire. Pour Sorel, la grève et le mythe qui lui est lié concentrent toutes les composantes traumatiques pour la conscience bourgeoise; en ce sens la grève représente une sorte de superattraction sadique.

4. Voir à ce sujet Oksana Boulgakova, "Sergueï Eisenstein et son Berlin psychologique - entre la psychanalyse et la psychologie structurale" (Kinovedtcheskie zapiski, $\mathrm{n}^{\circ} 2,1988$, p. 174-191).

5. Rank et Sachs parlent du «lien associatif fort» (p. 102) entre les éléments conscients et inconscients. La présence de ce lien ouvre la voie pour une décharge énergétique puissante qui se trouve être la base du plaisir esthétique.

6. La proximité de la sublimation du narcissisme en tant que stade non différencié des débuts de la sexualité infantile est examinée par Freud dans «Zur Einführung des Narzissmus" [Pour introduire au narcissisme] (1914). Voir "On Narcissism: An Introduction", dans S. Freud, General Psychological Theory (New York: Collier Books, 1963, p. 74-75).

7. "La chose la plus horrible dont je souffre, c'est la maladie de la volonté..." (Eisenstein, 1997/1998, p. 234).

8. Le diagnostic d'Eisenstein doit se lire sur le fond du fétichisme de son père, qui avait créé tout un dispositif de rangement d'une quantité énorme de chaussures noires laquées:

«Mon petit papa ne portait que les chaussures noires non pointues.

Il n'en existait pas d'autres.

Il en avait un assortiment énorme pour "toutes sortes d'occasions".

Il y avait un placard spécial pour les chaussures distinguées par leurs signes particuliers: "nouvelles", "anciennes", "avec une rayure".

De temps en temps la révision et le contrôle des chaussures avaient lieu." (1997, vol. 2, p. 83).

9. La traduction française des extraits de Rayon et Gnôle cités par l'auteur du présent article ne concorde pas toujours avec la version française de ce texte, qui paraît ici même, ailleurs dans ce numéro. Dans un souci de respect du travail des traducteurs, et pour éviter tout risque de teinter, de manière non souhaitée par lui, les propos de l'auteur du présent texte, nous avons jugé utile de passer outre à cette disparité, en fournissant en note l'autre version française du texte. Ici: "[...] je ne peux pas ne pas relever le matériau beaucoup plus "périlleux" qui relève du fétichisme primitif: la prééminence du pied et de la chaussure pointue, sous la bannière duquel on peut ranger tant Le Rayon que West. Sans parler des jambes inoubliables de Khokhlova, "vis morrix* " de ces ouvres [...]" [NdÉ].

10. Autre version française du même texte, ailleurs dans ce numéro: «Room souffre d'une névrose - je ne sais pas précisément comment la science la désigne - qui lui fait trouver du plaisir dans ce qui est répugnant, principalement en rapport avec le processus de prise ou de rejet de nourriture (nourriture "dégoûtante", vomissements, souillures à l'aide de nourriture, etc.) et avec la laideur sous l'aspect d'une transgression physique des normes, en particulier dans le domaine de la "texture " (de la peau et autres tissus - gourme, difformités, maladies de peau, vieilles hardes en lambeaux, pouilleuses, tissus vestimentaires eux-mêmes lépreux)" [ $\mathrm{NdÉ}]$. 
11. Autre version française du même texte, ailleurs dans ce numéro: "Room, séduit par la proximité et l'abondance d'un matériau pour lui troublant, collecté soi-disant pour des motifs utilitaires, au lieu de le soumettre au processus de sublimation et à la tâche qu'il s'est fixée, a au contraire succombé à la tentation et suivi une autre voie: celle de la satisfaction de sa névrose dans les formes du film qu’il était en train de créer, au lieu de dépasser ce principe névrotique. Et c'est là que réside la tragédie" [ $\mathrm{NdÉ}]$.

12. Autre version française du même texte, ailleurs dans ce numéro: «La puissance et la portée de leurs œuvres s'effectuent au détriment de défauts énormes qui altèrent leur fondement nerveux. L'aspect formel de leurs œuvres se caractérise par une forme manifeste de sadisme sublimé, ce qui les rend incomparables par l'effet bouleversant qu'elles produisent. La cruauté et l'implacabilité - le sadisme est la principale névrose indispensable à un réalisateur en général: sous le rapport des produits sublimés du sadisme, le réalisateur est comparable au chirurgien et... au boucher " [NdÉ].

13. Georges Didi-Huberman a remarqué avec justesse que le sadisme chez Eisenstein prend la forme de la rupture, de l'intervalle d'un souffle d'horreur, et l'image fait comme si elle s'efforçait de vaincre la figuration et d'obtenir "la possibilité formelle de transgresser la forme" (La Ressemblance informe, Paris: Macula, 1995, p. 326-333).

14. Voir là-dessus également l'ouvrage de Jean Laplanche, Vie et mort en psychanalyse (Paris: Gallimard, 1970, p. 143).

15. Ce film pourrait être La Vengeance du forgeron (scénario André Heuzé, Pathé, 1907), quoique le résumé qu'en donne le Kinematograph and Lantern Weekly n ${ }^{\circ} 18$ (12.09.07) differe quelque peu de celui d'Eisenstein (l'épouse du forgeron trompe celui-ci avec un châtelain et lors de sa revanche, le forgeron se bat en duel dans la forge avec son rival à l'aide de lourds marteaux. Ce qui laisse la place pour une blessure au fer rouge sans pour autant en faire le "clou " de la scène...) [remerciements à Henri Bousquet pour son aide] [NdÉ].

16. Cette instabilité de rapport entre bourreau et victime est décrite par René Girard comme un des traits fondamentaux du sadisme: "Le sadique ne peut pas se donner l'illusion qu'il est le médiateur sans transformer sa victime en un autre lui-même. Au moment même où il redouble de brutalité, il ne peut pas s'empêcher de se reconnaître dans l'Autre souffrant. Tel est le sens profond de cette étrange "communion" si souvent observée entre la victime et le bourreau. " (Mensonge romantique et vérité romanesque, Paris: Grasset, 1961, p. 212-213).

17. Eisenstein se souvient d'une passion semblable, pour des "dessins découpés", "dont les parties étaient maintenues ensemble au moyen d'un système de fines cordelettes. Combien de ces dessins à ramages ai-je décalqués dans les journaux illustrés de maman! Combien en ai-je plus tard combiné ou composé moi-même!» (1978b, p. 168).

18. Eisenstein évoque ainsi le travail du graphologue allemand Raphaël Scherman: "Scherman [...] décrit dans ses livres les contours des objets qu'il déchiffre dans le dessin de la signature et qui lui servent de clef pour déceler les "complexes" psychiques fondamentaux de ses patients" (1978b, p. 253).

19. Gilles Deleuze, en développant ces idées de Klein, a montré que le processus de symbolisation est basé sur le mouvement entre la profondeur indifférenciée et la surface et peut être décrit comme un des stades de la formation du sens qui se sépare de l'objet et de l'image "superficielle". Selon Deleuze, il y a une certaine doublure de l'« image", qui se projette sur un "double écran": "On dirait que l'action tout entière s'est projetée sur un double écran, l'un constitué par la surface sexuelle et physique, l'autre, par une surface déjà métaphysique ou "cérébrale" (Logique du sens, Paris: Minuit, 1969, p. 242). En tant que modèle du "double écran", Deleuze utilise l'image de la membrane qui sépare le dedans et le dehors chez. Freud dans $A u$-delà $d u$ principe du plaisir. 
20. Le texte original allemand et sa traduction française se trouvent dans François Albera, Eisenstein et le constructivisme russe. "Stuttgart", dramaturgie de la forme (Lausanne: L'Âge d'Homme, 1990, p. 37 et 67).

21. "Introduction et compléments à [Stuttgart]" dans François Albera, op. cit., p. 96-97.

\section{OUVRAGES CITÉS}

N.B. les ouvrages cités d'Eisenstein se retrouvent, en fin de numéro, dans une liste particulière.

Albera, François. Eisenstein et le constructivisme russe. "Stuttgart", dramaturgie de la forme. Lausanne: L'Âge d'Homme, 1990.

Benjamin, Walter. Selected Writings. Vol. 1. Cambrigde (Mass.) \& London: Belknap Press, 1996.

Freud, Sigmund. Trois essais sur la théorie de la sexualité. Paris: Gallimard, 1962.

Freud, Sigmund. "Instincts and Their Vicissitudes» et "On Narcissism: An Introduction" dans S. Freud, General Psychological Theory. New York: Collier Books, 1963a.

Freud, Sigmund. «Fetishism " dans S. Freud, Sexuality and the Psychology of Love. New York: Collier Books, 1963b.

Freud, Sigmund. Un Souvenir d'enfance de Léonard de Vinci. Paris: Gallimard, [1927] 1973.

Jones, Ernest. The Life and Work of Sigmund Freud. New York: Basic Books, 1957.

Klein, Melanie. "An Obsessional Nevrosis in a Six-Year Old Girl" dans M. Klein, The Psychoanalysis of Children, New York: Grove Press, 1960 [trad. franç. : La Psychanalyse des enfants. Paris: PUF, 1959].

Klein, Melanie. "The Importance of Symbol-Formation in the Development of the Ego" dans M. Klein, Love, Guilt and Reparation and Other Works 1921-1945. New York: The Free Press, 1984.

Rank, Otto et Hans Sachs. "The Significance of Psychoanalysis for the Humanities" dans American Imago, vol. 21, Spring-Summer, $n^{\text {os }}$ 1-2 (1964) [trad. franç.: Psychanalyse et sciences humaines. Paris: Presses universitaires de France, 1980.].

La sublimation comme formation de la forme. Note sur un article inédit d'Eisenstein 


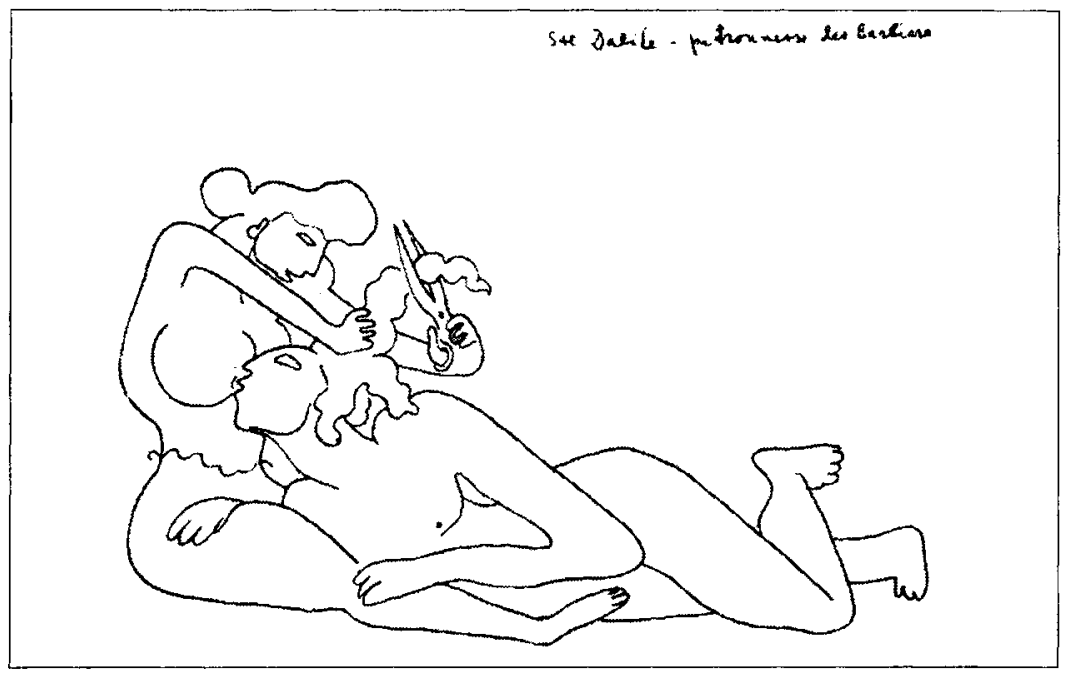

S. M. Eisenstein, Ste Dalile - patronnesse des barbiers [du cycle Samson et Dalila], vers 1931

Dimensions : $27 \times 37,3 \mathrm{~cm}$

Médium: encre, papier

Coll. : Archives d'État russes de la littérature et de l'art

Photographie: Alexander Sorin

(C) fondation Daniel Langlois 